\title{
Malaria vaccine wows and seeds COVID-19 vaccine effort
}

\author{
A malaria vaccine not only has achieved record-setting protection in a large trial, but also spurred the UK's \\ COVID-19 vaccine effort.
}

A

large phase- 3 trial, launched last month, will test a malaria vaccine that is the best hope yet of generating immunity to a disease that kills over 400,000 people a year and hits young children the hardest. At the helm of the effort are some of the University of Oxford developers behind one of the world's most widely used COVID-19 vaccines: Vaxzevria/Covishield.

The four-country malaria trial, which started on 10 May, comes on the back of published phase 2 results demonstrating up to $77 \%$ efficacy for the vaccine, called R21, when assessed for one year in 450 children in Burkina Faso. "This is the highest efficacy we have ever seen in any malaria vaccine candidate," says Halidou Tinto, a parasitologist at the Health Sciences Research Institute in Nanoro and a lead author on the study. "To be honest, it was a surprise."

No vaccine against a eukaryotic parasite has been approved for human use. Before $\mathrm{R} 21$, the record holder was the RTS,S vaccine (Mosquirix), which is made by GlaxoSmithKline and achieved 56\% efficacy over one year. RTS,S is now being deployed in Malawi, Kenya and Ghana as part of a pilot program to further gauge safety, efficacy and logistics.

Both vaccines, R21 and RTS,S, induce immune responses using the same antigen: a piece of the circumsporozoite protein that decorates the outside of the malaria parasite Plasmodium falciparum at the sporozoite stage. The parasite is in that sporozoite stage when it first enters the human body from its mosquito vector, and the hope is that the vaccines will trigger an overwhelming immune response that neutralizes the parasite before it invades liver cells and then human blood cells, where it causes symptoms.

Although researchers have pursued other malaria antigens, the circumsporozoite protein has so far been the most effective. In GlaxoSmithKline's RTS,S, which was developed in the 1980s, the immunogen is a fusion of the $\mathrm{C}$ terminus and central repeat region of the circumsporozoite protein with the hepatitis B surface antigen (HBsAg). When expressed in yeast, the fusion protein assembles into an immunogenic, virus-like

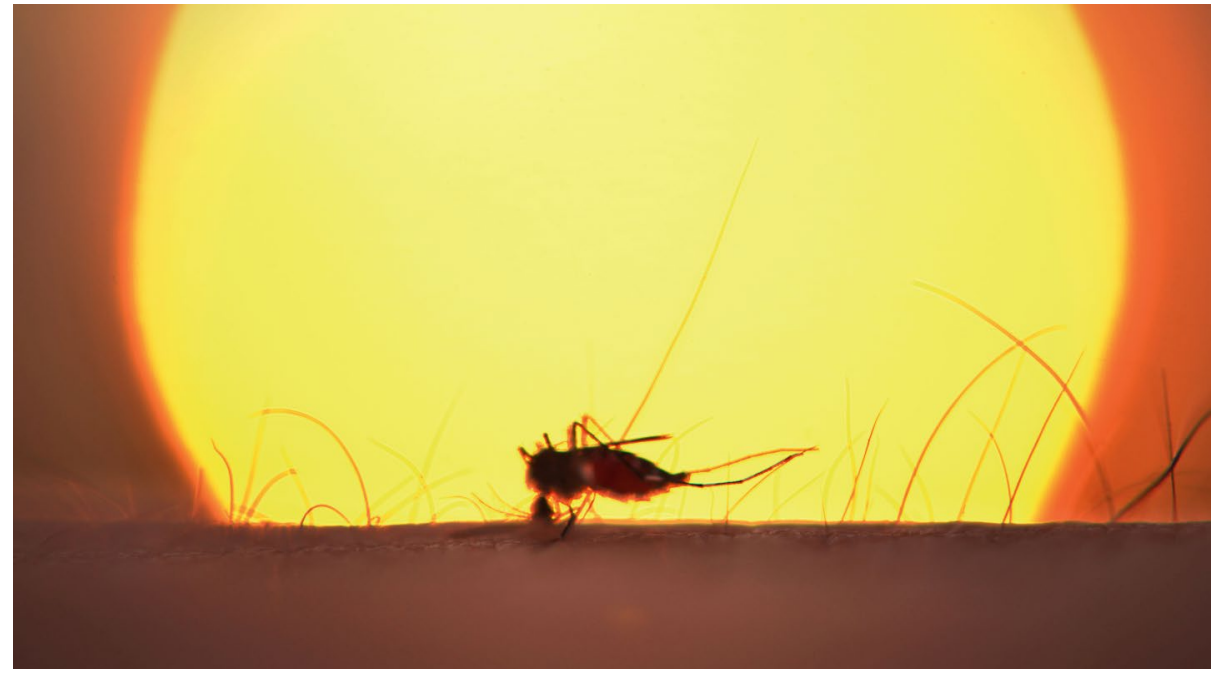

A mosquito takes a blood feed. Scientists are optimistic about the R21 malaria vaccine, the first to meet the WHO's 75\% protection target. Credit: Apichart Meesri / Alamy Stock Photo

particle. But the assembly requires free HBsAg, and the resulting vaccine contains substantial amounts of both the malaria and the hepatitis B antigens.

Scientists at the University of Oxford's Jenner Institute, led by vaccinologist Adrian Hill, saw an opportunity for improvement. Because the hepatitis B antigens in RTS,S meant the circumsporozoite fusion protein comprise only $20 \%$ of the vaccine, Hill and his colleagues reasoned that eliminating the free HBsAg in the vaccine would boost the amount of circumsporozoite protein-and power the immune response to it. The team developed a new method to do so, shifting the protein's production from Saccharomyces cerevisiae to a different yeast, Pichia pastoris, and using a different genetic promoter to drive expression.

The vaccine adjuvant also changed. GlaxoSmithKline combines RTS,S with an adjuvant the company has developed that consists of two components: a saponin and a lipopolysaccharide derivative that acts as a toll-like receptor 4 (TLR4) agonist, activating the innate immune system. R21's adjuvant-called Matrix-M and made by Novavax-contains a different saponin and lacks the TLR4 agonist. (Matrix-M is also in Novavax's protein-based COVID-19 vaccine,
NVX-CoV2373.) “The new adjuvant is easier to manufacture, which will probably bring down the cost," says Kwadwo Koram, an epidemiologist at the University of Ghana in Accra.

The University of Oxford and Novavax have partnered with the Serum Institute of India to produce 200 million doses of R21 a year, if it is eventually approved by regulators. This relationship with the Serum Institute, forged to produce malaria vaccines, was instrumental in facilitating a pandemic collaboration to produce another vaccine developed in part by Hill and his colleagues: the Oxford-AstraZeneca vaccine against COVID-19, ChAdOx1 nCOV-19, sold under brand names Vaxzevria and Covishield. "It was an important connection," says Hill. "It allowed them to invest, at risk, in trying to make hundreds of millions of doses last year, before we had efficacy data." About 500 million doses of the AstraZeneca vaccine have now been distributed globally, says Hill, most of them produced by the Serum Institute.

The team's work on malaria vaccines also helped to lay the technological groundwork for Vaxzevria. The COVID-19 vaccine relies on a chimpanzee adenoviral vector (ChAdOx1) that was developed from a vector 
(ChAd63) the Oxford team previously worked with for an experimental malaria vaccine. The aim was to deliver circumsporozoite using ChAd63 to prime the immune system and then to use a circumsporozoite-modified vaccinia virus Ankara as a booster.

Chimpanzee virus was attractive, says Hill, because the team was mindful that antibody titers against potential human adenoviral vectors were high in Africa. At the time, they were targeting a malaria protein called thrombospondin-related anonymous protein (TRAP), which is important for the parasite's invasion of the liver. To fight malaria in the liver, they needed to rouse $\mathrm{T}$ cells rather than antibody production, and early data suggested that the chimpanzee adenoviral vector would be well suited to that. Ultimately, the circumsporozoite protein proved a more effective antigen.

Overall, R21 still has obstacles to achieving regulatory approval and large-scale rollout. "The natural history of most of these studies is that the protective efficacy declines with a larger study and longer follow up," says Nicholas White, who studies tropical medicine at Mahidol University in Bangkok.

Testing R21 in different countries could prove critical to establishing its potential effectiveness. The phase 2 trial, a double-blind, randomized, placebo-controlled study, was held in Burkina Faso, which has an unusually strong seasonal pattern of malaria incidence. Most of the participants were given the vaccine shortly before the rainy season, when malaria is more prevalent. That means that most of the challenges to their immunity occurred when responses to the vaccine were still high, says White. "If you have a vaccine that doesn't last very long, then you're stacking the cards in your favor if you have a high attack rate early on because that's when you'll expect to see protection," he says. The phase 3 trial will add sites in Kenya, Mali and Tanzania. Data from Kenya and Tanzania will be particularly critical, says Tinto, because malaria is not seasonal there.

It will also be important to follow participants for several years, says Stefan Kappe, who studies malaria parasite biology and immunology at the Seattle Children's Research Institute in Washington. For effective deployment in Africa, it would be best to have a vaccine that provides durable immunity lasting three or more years, he says, to reduce the need for extra inoculations.

That is a high bar indeed. With RTS,S, efficacy declined to about $17 \%$ over about four years, even when one booster shot was given. "For RTS,S, the main limitation is the durability," says Carlota Dobaño, an infectious disease immunologist at the
Barcelona Institute for Global Health. "The effects of the vaccine don't last long, and we don't know why."

The World Health Organization has set a vaccine target of $75 \%$ protection over at least one year to merit an investment in large-scale deployment. Although other malaria vaccine approaches are in development, R21 is the first to meet that target.

Some researchers, including those at the biotech company Sanaria, are testing ways to vaccinate using full, non-replicating parasites-a challenging approach that requires maintaining aseptic mosquitoes to serve as hosts. The company has been laboring to perfect its vaccine for nearly two decades: "Nobody has manufactured a vaccine in mosquitoes before," says Stephen Hoffman, Sanaria's co-founder and chief executive. Sanaria has been irradiating sporozoite-stage parasites to disable them before injection, but is now exploring chemical ablation and gene editing of Plasmodium, the latter as a collaboration with Kappe, to render them harmless. One advantage of this whole-parasite method is that it would expose vaccine recipients to a broader swath of the parasite's proteins, potentially boosting the intensity and diversity of immune responses.

In the long term, Hill hopes that R21 could be combined with other malaria vaccines, still in development, to provide a broader and more powerful immune response. There is some worrying sequence variability in the circumsporozite protein that could weaken protection by giving rise to escape mutations, says Alyssa Barry, a genomic epidemiologist at Deakin University and the Burnet Institute in Melbourne, Australia. "Potentially there are many different strains circulating even within a single village," she says.

For RTS,S, there is already some evidence that such variants have reduced efficacy, Barry says. Researchers will want to know whether the same could be happening with R21, and whether vaccines could be improved by incorporating multiple circumsporozoite protein sequence variants.

Ultimately, even if $\mathrm{R} 21$ proves successful, the shots alone are unlikely to be sufficient to defeat malaria, says Koram; mosquito control practices, for example, will remain critical. "For malaria, we are not going to have one product that will do everything," he says. "If we have a vaccine and we're not behaving properly, we'll still have breakthrough infections."

\section{Heidi Ledford \\ London, UK}

Published online: 13 July 2021

https://doi.org/10.1038/s41587-021-00983-8

\section{Germany's biotech behemoth}

In June, stalwart German biotech MorphoSys cemented its future as a commercial-stage company through the $\$ 1.7$ billion acquisition of US epigenetics company Constellation Pharmaceuticals and an agreement with Royalty Pharma to finance the takeover. The deal with its US rival sees the 29-year-old German company gain two advanced epigenetic product candidates: the BET inhibitor pelabresib, in phase 3 testing to treat myelofibrosis; and CPI-0209, an EZH2 inhibitor, which is in a phase 2 trial to treat hematologic malignancies and solid tumors. MorphoSys's HuCal (Human Combinatorial Antibody Library) technology platform led to the discovery of Tremfya (guselkumab), an anti-IL-23 monoclonal antibody developed by Johnson \& Johnson's Belgian subsidiary Janssen that was approved by the US Food and Drug Administration (FDA) in 2017 to treat psoriasis. In July 2020, MorphoSys, in partnership with Incyte, gained its first market go-ahead with FDA approval of Monjuvi (tafasitimab-cxix), in combination with lenalidomide, to treat adults with relapsed or refractory diffuse large B-cell lymphoma, the most common type of non-Hodgkin's lymphoma. Monjuvi is a humanized cytolytic CD19-targeting IgG1/2 hybrid monoclonal antibody with an engineered $\mathrm{Fc}$ domain, which was licensed from Xencor. The modified Fc mediates B cell lysis through apoptosis and immune effector mechanisms through antibody-dependent cell-mediated cytotoxicity and antibody-dependent cellular phagocytosis.

MorphoSys, which is headquartered in Planegg, near Munich, has more than 100 products in its wider pipeline, many of them partnered, and those in its proprietary pipeline focus on cancer and autoimmune diseases. The Royalty Pharma deal gives MorphoSys $\$ 1.4$ billion up front to fund its growth, in exchange for royalties on three MorphoSys programs partnered with pharma: Tremfya, otilimab and the Alzheimer's disease candidate gantenerumab. The controversial green light for Biogen's Alzheimer's disease drug aducanumab was seen to set an advantageous precedent for others pursuing programs in neurodegenerative diseases, resulting in a boost to share value for MorphoSys from $\$ 0.81$ to $\$ 21.64$.

Published online: 13 July 2021

https://doi.org/10.1038/s41587-021-00990-9 